\title{
Video Article \\ Behavioral Disturbances: An Innovative Approach to Monitor the Modulatory Effects of a Nutraceutical Diet
}

\author{
Alessandro Di Cerbo* ${ }^{1}$, Sara Sechi* ${ }^{2}$, Sergio Canello ${ }^{3}$, Gianandrea Guidetti ${ }^{3}$, Filippo Fiore ${ }^{2}$, Raffaella Cocco ${ }^{2}$ \\ ${ }^{1}$ School of Specialization in Clinical Biochemistry, "G. d'Annunzio" University \\ ${ }^{2}$ Department of Veterinary Medicine, Pathology and Veterinary Clinic Section, University of Sassari \\ ${ }^{3}$ Research and Development Department, Forza10 USA Corp. \\ * These authors contributed equally
}

Correspondence to: Alessandro Di Cerbo at alessandro811@hotmail.it

URL: https://www.jove.com/video/54878

DOI: doi: $10.3791 / 54878$

Keywords: Behavior, Issue 119, behavioral disturbances, specific diet, intense and restless activity, specific sensor, clinical symptoms, behavioral symptoms

Date Published: 1/3/2017

Citation: Di Cerbo, A., Sechi, S., Canello, S., Guidetti, G., Fiore, F., Cocco, R. Behavioral Disturbances: An Innovative Approach to Monitor the Modulatory Effects of a Nutraceutical Diet. J. Vis. Exp. (119), e54878, doi:10.3791/54878 (2017).

\section{Abstract}

In dogs, diets are often used to modulate behavioral disturbances related to chronic anxiety and stress caused by intense and restless activity. However, the traditional ways to monitor behavioral changes in dogs are complicated and not efficient. In the current clinical evaluation, a new, simple monitoring system was used to assess the effectiveness of a specific diet in positively modulating the intense and restless activity of 24 dogs of different ages and breeds. This protocol describes how to easily and rapidly evaluate improvement in a set of symptoms related to generalized anxiety by using a specific sensor, a mobile phone app, a wireless router, and a computer. The results showed that dogs treated with specific diets showed significant improvement in the times spent active and at rest after 10 days $(p<0.01$ and $p<0.05$, respectively). These dogs also showed an overall significant improvement in clinical and behavioral symptoms. A specific sensor, along with its related hardware, was demonstrated to successfully monitor behavioral changes relating to movement in dogs.

\section{Video Link}

The video component of this article can be found at https://www.jove.com/video/54878/

\section{Introduction}

Dogs are generally recognized as the most devoted domesticated animals that live with humans. They are often considered to be family members whose behavioral changes are regarded as serious concerns, especially when these changes threaten their physical integrity and general wellbeing ${ }^{1}$. Therefore, the development of adjuvant approaches to common therapies for relieving canine behavioral disturbances would help families to improve the quality of life of their dogs, avoiding unwanted phenomena such as dog abandonment and euthanasia ${ }^{2}$. Most of these behavioral disturbances are related to anxiety caused by stress, and the anxiety may become pathological without proper intervention ${ }^{1,3}$.

It has been hypothesized that anxiety disorders in dogs are caused not only by significant life changes, but also by chronic or post-traumatic stresses that can alter their homeostasis and can consequently lead to adaptation disorders ${ }^{1,3}$. This study is based on a clinical evaluation of behavioral disturbances mainly ascribed to generalized anxiety. The typical clinical symptoms of this disorder include constant or increasing reactivity, body and environmental exploration, activation, alertness, and excessive barking; it also often affects the social interactions between dog and owner ${ }^{1,4,5}$. The predisposing factors could be intrinsic, such as genetics, or extrinsic, such as environmental stimuli ${ }^{1,6}$. In fact, the aforementioned clinical symptoms can become frequent even without a triggering environmental stimulus. In this sense, the study of the origin of these factors becomes essential for proper diagnosis and subsequent therapy.

The most common therapies for generalized anxiety rely on counterconditioning and desensitization techniques, where the dog learns how to behave once faced with a stimulus that causes anxiety, or on a pharmacological approach based on anxiolytic drug administration ${ }^{7}$. Based on these considerations, 24 dogs affected by behavioral disturbances mainly ascribed to generalized anxiety received a counterconditioning and desensitization behavioral therapy combined with a nutraceutical diet for 10 days. The diet consisted of a mixed formula of fish proteins, rice carbohydrates, Punica granatum, Valeriana officinalis, Rosmarinus officinalis, Tilia spp, tea extract, and L-tryptophan, with an omega-3:-6 ratio of 1:0.8. Literature reports clearly evidenced that $P$. granatum is used to treat chronic anxiety and insomnia ${ }^{1,8}$, while Valeriana officinalis is used for mild sleep disorders and nervous tension ${ }^{9,10}$. Furthermore, anti-anxiety and anti-depressant effects have been observed after Rosmarinus officinalis $^{11-14}$ and Tilia $\operatorname{spp}^{15,16}$ consumption. L-theanine, one of the tea constituents, has been shown to play a role in reducing stress and decreasing heart rate in chronic anxiety ${ }^{17,18}$. Conversely, many studies reported the onset of anxiety, mood, and depressive symptoms after Ltryptophan depletion and/or an omega-3 deficiency ${ }^{19,20}$. 
Generalized anxiety behavior and clinical symptoms, including marking, anxiety, diffidence, irregular biorhythm, reactivity, activation, irritability, alertness, environmental exploration, body exploration, attention requirement, dandruff, itchiness, flush, seborrhea, fur opacity, vomiting, diarrhea, flatulence, lachrymation, and anal sac repletion, were also evaluated. Most of these symptoms were accompanied by signs that induced dogs to spend more time awake and active, rather than at rest or asleep. Therefore, activity and rest times spent by each dog before and after evaluation were assessed. To continuously monitor daily improvements of activity and rest time, a commercially available sensor, which was fixed to the collars of the dogs and connected to a mobile phone or to a Wi-Fi station, was used.

\section{Protocol}

The protocol was examined and approved by the Veterinary Ethical Review Committee prior to the beginning of the study. The recommendations of the ARRIVE guidelines in animal research were also consulted and considered ${ }^{21-25}$.

\section{Dog Selection and Food Supplementation}

1. Select 24 dogs of different breeds (mean age and weight \pm SEM: $2.9 \pm 0.3$ years and $32.01 \pm 1.17 \mathrm{~kg} ; 14$ males, 10 females) with evident clinical symptoms of behavioral disturbances, such as anxiety, diffidence, irregular biorhythm, reactivity, activation, irritability, alertness, and constant environmental exploration.

2. Randomly divide the animals into two groups and place each into a $215,278 \mathrm{sq} f \mathrm{ft}$ single box. Following the manufacturer's instructions, give the appropriate dose of standard diet $(S D, n=12)$ or nutraceutical diet $(N D, n=12)$ for 10 days, according to the weights of the animals (Table 1).

3. Complete two veterinary inspections of the dogs before (T0) and 10 days after (T1) the treatment.

\section{Behavior Symptoms Acquiring and Scoring}

1. Have a certified veterinary behaviorist score the behavioral (marking, anxiety, diffidence, irregular biorhythm, reactivity, activation, irritability, alertness, environmental exploration and attention requirement) and clinical (dandruff, itchiness, flush, seborrhea, fur opacity, vomiting, diarrhea, flatulence, lachrymation and anal sac repletion) conditions of each dog.

1. For each dog, gather a score before and after the 10-days evaluation as follows: $1=$ absence of symptom; $2=$ moderate presence of symptoms; 3 = marked presence of symptoms.

2. At the end of the evaluation summarize, for each symptom, the scores of the dogs of each group before and after 10 days. Plot the data on a statistical software.

\section{The Sensor}

Note: The sensor has a 3-axis accelerometer, allowing it to gather movement in each direction $(\mathrm{x}, \mathrm{y}, \mathrm{z})$. It weighs $8 \mathrm{~g}$ and is extremely small (41 $x 28 \times 11 \mathrm{~mm}$ ). Moreover, it is compatible with every mobile device that features a global wireless communication. It has a rechargeable battery that can last for 14 days after charging. The data output is calculated in points, which are the units of time spent active and at rest by each dog during the day.

1. Ensure that the collar is not wider than $30 \mathrm{~mm}$ for the optimal fit of the sensor.

2. Using the procedure outlined in sections 3 and 4 , evaluate the behavioral changes related to the time spent active and at rest before and after treatment with the specific diet.

\section{Sensor Setting}

1. Open the micro USB cap on the bottom of the unit and use the cable provided to connect the sensor to the computer's USB $1 \mathrm{x} / 2.0$ port or to a Class 2/Limited power supply with a USB output. When an LED starts blinking, charge the sensor for at least 90 min.

2. Download and install the dedicated free mobile app from the web store.

\section{Activity Monitoring and Analysis}

1. Insert the plugs of a wireless router and the dedicated Wi-Fi base station into two different sockets. Wait until the router is ready and the Wi-Fi base station starts flashing.

2. Enable Bluetooth on the mobile device and make sure that it is connected to the Internet.

3. Open the app and sign up. Then, tap on "Add new dog" and follow the steps below.

1. Take a picture of the dog and provide its name.

2. Appropriately set the gender, age, weight, neutering status, and location of the dog.

3. Set the primary and the secondary breed of the dog.

4. Select, if applicable, the presence of allergies (skin, ear, etc.), arthritis, brain aging, cancer, diabetes, heart disease, or excessive weight.

5. Select one of the three available lifestyles, with their respective points, according to personal requirements (1. Average, 2. Active, or 3. Olympian).

NOTE: Depending on the age of the dog, the points of each lifestyle will change. Given that the aim of this evaluation was to decrease the hyperactivity and the stress of the dogs, the first lifestyle, "Average," was set as the final goal to reach. 
6. Tap on "Wi-Fi base station" and then on "Pair a base station" to connect the sensor to the Wi-Fi base station. Wait until the word "FitBark" appears below "Pair a base station."

4. Clip a charged sensor to the collar of the dog.

5. Repeat steps 4.3 to 4.4 for each dog.

\section{Bark Recording}

1. Fix a digital voice recorder to the wall of each box at the beginning of the study.

2. Start recording the bark activity.

1. Every day, before acquiring new data, connect the digital voice recorder to a computer by means of a retractable USB connector provided along with the recorder.

2. Drag the folder with voice data from the device to the computer and rename it with the current date.

3. Repeat steps 6.2.1 - 6.2.2 every day for 10 days.

4. At the end of the evaluation transform (in s) the bark time recorded. Summarize the bark time for each group before and after 10 days. Plot the data on a statistical software.

\section{Representative Results}

Table 1 shows the daily amount of nutraceutical diet suggested by the manufacturer. In Figure 1, the daily mean activity and rest times spent by dogs belonging to the SD and ND groups during the evaluation period are shown. For instance, a significant decrease from a T0 value of 7,343 \pm 611.7 to a T1 value of $5,093 \pm 526.5$ was observed in the ND group after 10 days, while no significant difference was observed in the SD group (Figure 1A, ${ }^{*} p<0.05$ ). Conversely, the daily mean rest time significantly increased from a T0 value of $7.6 \pm 0.3 \mathrm{~h}$ to a T1 value of $9.5 \pm 0.3 \mathrm{~h}$ in the ND group after 10 days $\left({ }^{* *} p<0.01\right)$, while no significant difference was observed in the SD group (Figure 1B).

Regarding behavioral symptoms, a reduction of the mean intensity of marking, from 2.50 to 2.41, was observed in dogs belonging to ND group, while no difference was observed in those belonging to the SD group (Figure 2A). On the contrary, anxiety, diffidence, and irregular biorhythm showed a significant reduction in dogs belonging to the ND group, from a T0 value of $2.50 \pm 0.19$ to a T1 value of $1.16 \pm 0.11$ ( ${ }^{* \star *} p<0.001$, Figure 2B), from a T0 value of $2.08 \pm 0.28$ to a T1 value of $1.17 \pm 0.12$ (Figure $2 \mathrm{C},{ }^{*} p<0.05$ ), and from a T0 value of $2.08 \pm 0.28$ to a T1 value of $1.08 \pm 0.08$ (Figure 2D, ${ }^{* *} p<0.01$ ), respectively. No significant difference was observed in the respective SD groups. Also, reactivity, activation, irritability, alertness, environmental exploration, and attention requirement mean intensities showed significant reductions after ND supplementation. In particular, reactivity decreased from a T0 value of $2.16 \pm 0.27$ to a T1 value of $1.25 \pm 0.13$ (Figure $2 \mathrm{E}$, ${ }^{* *} p<0.01$ ), activation decreased from a T0 value of $2.25 \pm 0.25$ to a T1 value of $1.33 \pm 0.14$ (Figure $2 F,{ }^{*} p<0.05$ ), irritability decreased from a T0 value of $2.66 \pm 0.18$ to a T1 value of $1.66 \pm 0.22$ (Figure $2 \mathbf{G},{ }^{* *} p<0.01$ ), alertness decreased from a T0 value of $2.50 \pm 0.19$ to a T1 value of $1.66 \pm 0.22$ (Figure $2 \mathrm{H}$, ${ }^{*} p<0.05$ ), environmental exploration decreased from a T0 value of $2.33 \pm 0.18$ to a T1 value of $1.66 \pm 0.22$ (Figure 2I, ${ }^{* *} p<0.01$ ), and attention requirement decreased from a T0 value of $2.24 \pm 0.15$ to a T1 value of $1.55 \pm 0.21$ (Figure $2 \mathbf{J},{ }^{*} p<0.05$ ). No significant difference was observed in the respective SD group.

In Figure 3, the mean intensities of clinical symptoms in dogs belonging to the SD and ND groups, before (T0) and after the 10-day evaluation period (T1), are shown. Dandruff significantly decreased from a T0 value of $2.33 \pm 0.14$ to a T1 value of $1.08 \pm 0.08$ (Figure $3 A$, *** $p<0.001$ ). Also, itchiness, flush, seborrhea, and fur opacity significantly decreased from a T0 value of $2.08 \pm 0.26$ to a T1 value of $1.04 \pm 0.05$, from a T0 value of $2.11 \pm 0.24$ to a T1 value of $1.16 \pm 0.11$, from a T0 value of $2.35 \pm 0.25$ to a T1 value of $1.33 \pm 0.14$, and from a T0 value of $2.22 \pm 0.13$ to a T1 value of $1.41 \pm 0.15$, respectively (Figure 3B-E, * $p<0.05$ ). A similar trend was observed for vomiting, diarrhea, flatulence, lachrymation, and anal sac repletion scores, which significantly decreased from a T0 value of $2.75 \pm 0.10$ to a T1 value of $1.58 \pm 0.17$ (Figure $3 \mathrm{~F},{ }^{*} p<0.001$ ), from a T0 value of $2.69 \pm 0.12$ to a T1 value of $2.06 \pm 0.19$ (Figure $3 G,{ }^{*} p<0.01$ ), from a T0 value of $1.75 \pm 0.13$ to a T1 value of $1.25 \pm 0.13$ (Figure $3 \mathrm{H},{ }^{*} p<0.05$ ), from a T0 value of $2.16 \pm 0.11$ to a T1 value of $1.32 \pm 0.03$ (Figure 3l, ${ }^{*} p<0.05$ ), and from a T0 value of $2.28 \pm 0.12$ to a T1 value of $1.30 \pm 0.14$ (Figure $3 \mathbf{J},{ }^{*} p<0.01$ ), respectively. No significant difference was observed in dogs belonging to the SD group.

Figure 4 shows the mean time spent in barking by dogs belonging to the SD and ND groups before (T0) and after the evaluation period (T1). A significant decrease from a T0 value of $180.21 \pm 15.35$ to a T1 value of $76.02 \pm 7.22$ was observed in the ND group after 10 days. No significant difference was observed in the SD group.

The data were analyzed using graphing and statistical software. All data are presented as \pm the standard error of the mean and were first checked for normality using the D'Agostino-Pearson normality test. Differences in activity, rest and barking time as well as symptoms during the evaluation period were analyzed using a two-way ANOVA test followed by Tukey's multiple comparisons test. $p<0.05$ was considered significant.

It is worth noting that, at the beginning of the study, each dog was automatically assigned by the mobile app software to achieve a desired daily activity according to weight, age, and breed. After treatment, all dogs belonging to the ND group showed a significant decrease in the mean daily activity $(p<0.05)$ with respect to SD group, which was even lower than expected, and a consequent significant increase in daily mean rest time $(p<0.01)$. These results were also well correlated with consequent clinical symptoms and signs (Figures 2 - 3 ), demonstrating significant improvement as well as a significant reduction in barking time (Figure $4, p<0.001$ ). Taken together, all these considerations strengthened the efficacy of the ND in improving the results of common behavioral therapies for generalized anxiety. These results are also in agreement with our recent paper, which described the efficacy of a similar diet in relieving some of the clinical symptoms, such as dandruff, itchiness, flush, diarrhea, and flatulence, that have all been taken into account in this study ${ }^{26}$.

It is also worth noting that clinical symptoms might also be the manifestation of an overall inflammation status, with a consequent oxidative stress imbalance. Inflammation is also known to contribute to the etiology of anxiety disorders, depression ${ }^{27}$, and neurotransmitter activity ${ }^{28}$. In this sense, we recently identified a specific compound, oxytetracycline, as a possible agent able to trigger an inflammatory condition both in 
vitro $^{29,30}$ and in vivo ${ }^{31,32}$. Oxytetracycline belongs to the class of tetracyclines, which are the most widely and legally used antibiotics in intensive farming (e.g., poultry ${ }^{30}$, livestock ${ }^{33}$, and aquaculture ${ }^{34}$ ) due to their low cost and high efficacy ${ }^{35}$. Unfortunately, oxytetracycline also has a high affinity for calcium-rich tissues, such as bone and teeth ${ }^{36}$, and can remain fixed in treated animals for extended periods, even respecting withdrawal time ${ }^{30}$. Moreover, pet food production relies on meat (mainly poultry) by-products, which are mechanically separated ${ }^{37}$. This kind of separation generates a bone-based meal bearing oxytetracycline residues that are present in commercially available diets (canned, semi-moist, and dry) at $20-30 \%$ and can accumulate within a pet's body.

Concerning the ND group, it is reasonable to hypothesize that the mean score intensity reduction of all clinical symptoms was thus a consequence of the anti-inflammatory and antioxidant effect of the nutraceutical substances Punica granatum ${ }^{38}$, Valeriana officinalis ${ }^{39}$, Rosmarinus officinalis ${ }^{40}$, Tilia $\mathrm{spp}^{41}$, tea extract ${ }^{42}$, and polyunsaturated fatty acids (PUFA) present within the diet. For instance, PUFA have been show to modulate behavioral symptoms in attention-deficit hyperactivity disorder (ADHD) patients and in aggressive dogs ${ }^{43}$. These dogs had lower docosahexaenoic acid (DHA) levels than normal, as well as a higher omega-6:omega-3 ratio ${ }^{44,45}$.
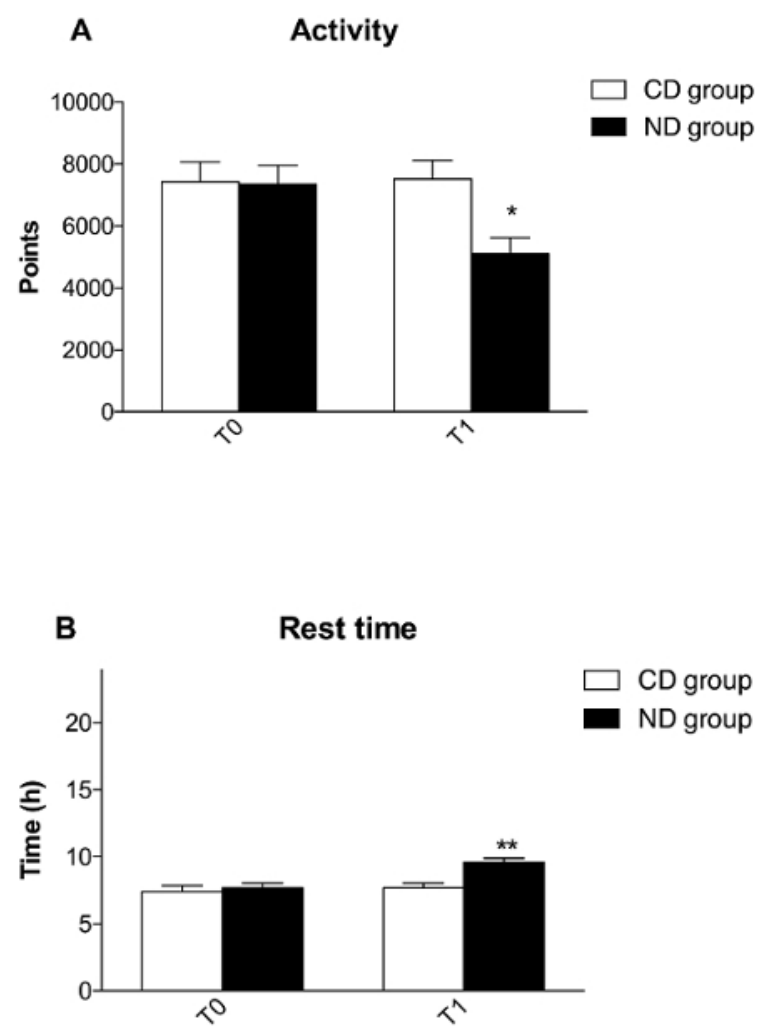

Figure 1. ND Reduces Activity Time and Increases Rest Time in Dogs. Schematic representation of the daily mean activity (A) and rest time (B) of dogs before (T0) and 10 days after (T1) SD and ND supplementation $\left({ }^{* *} p<0.01\right)$. The error bars are \pm the standard error of mean. Please click here to view a larger version of this figure. 

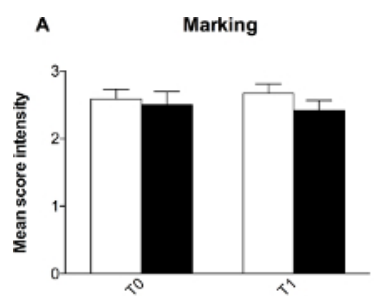

C Diffidence

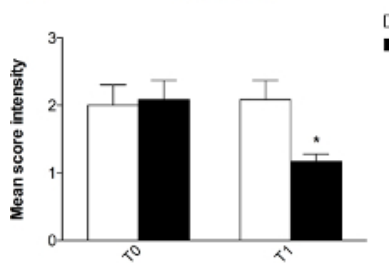

E Reactivity

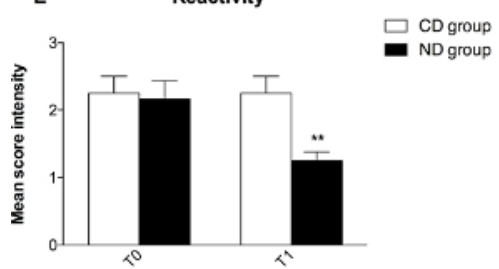

G Irritability
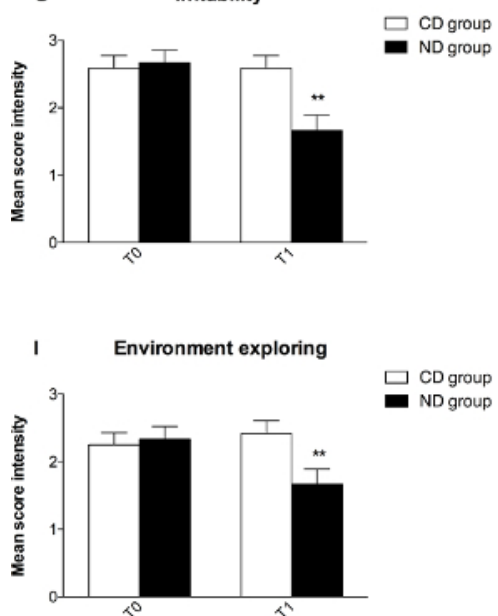

$$
\square \text { CD group }
$$

- ND group
B

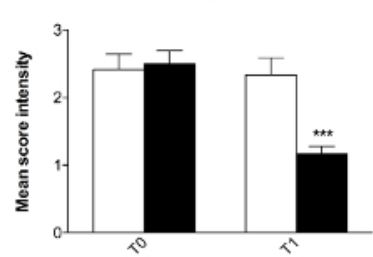

D Irregular biorythm
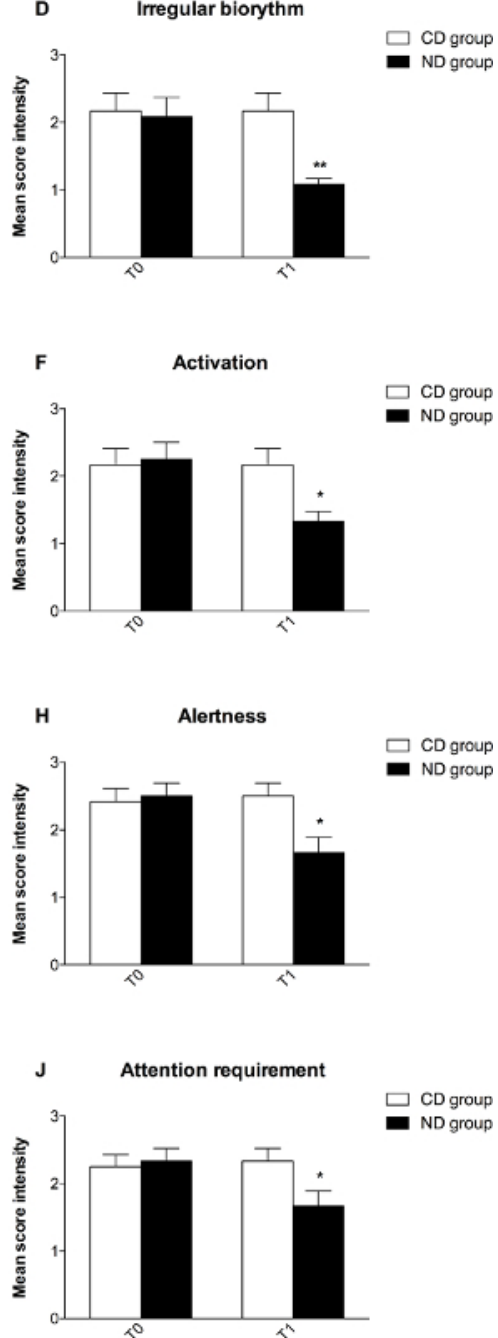

Figure 2. ND Improves the Behavioral Disturbances in Affected Dogs. Schematic representations of the mean score intensities of behavioral symptoms in dogs before (T0) and 10 days after (T1) SD and ND supplementation. (A) Marking. (B) Anxiety $\left({ }^{* * *} p<0.001\right)$. (C) Diffidence ( ${ }^{*} p$ $<0.05)$. (D) Irregular biorhythm $\left({ }^{* *} p<0.01\right)$. (E) Reactivity $\left({ }^{* *} p<0.01\right)$. (F) Activation $\left({ }^{*} p<0.05\right)$. (G) Irritability $\left({ }^{* *} p<0.01\right)$. (H) Alertness $\left({ }^{*} p<\right.$ $0.05)$. (I) Environmental exploration $\left({ }^{* *} p<0.01\right)$. (J) Attention requirement $\left({ }^{* *} p<0.01\right)$. The error bars are \pm the standard error of mean. Please click here to view a larger version of this figure. 

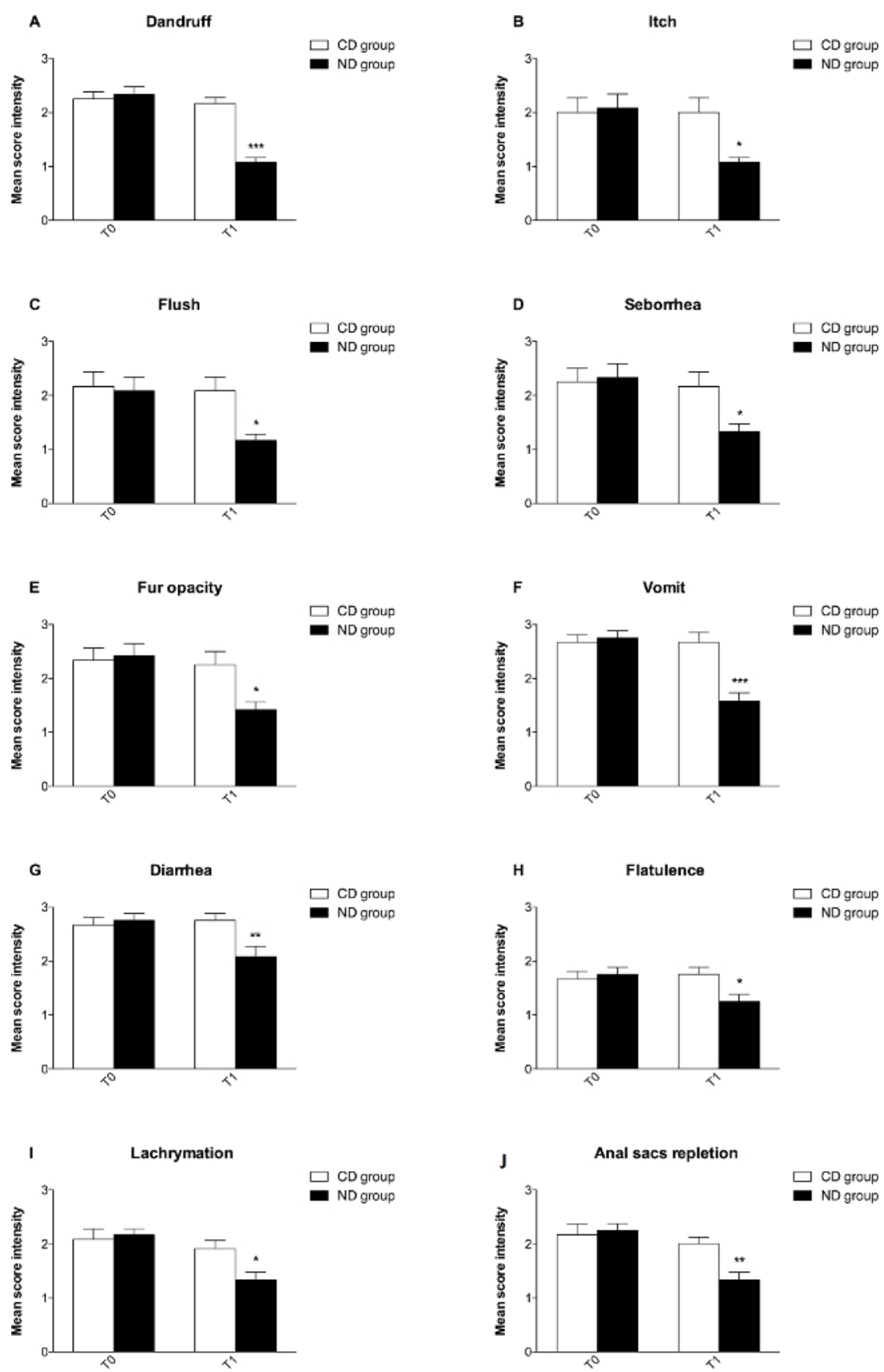

Figure 3. ND Improves the Clinical Signs in Affected Dogs. Schematic representation of the mean score intensities of clinical symptoms in dogs before (T0) and 10 days after (T1) SD and ND supplementation (A) Dandruff $\left({ }^{* * *} p<0.001\right)$. (B) Itch $\left({ }^{*} p<0.05\right)$. (C) Flush $\left({ }^{*} p<0.05\right)$. (D) Seborrhea $\left({ }^{*} p<0.05\right)$. (E) Fur opacity $\left({ }^{*} p<0.05\right)$. (F) Vomiting $\left({ }^{* * *} p<0.001\right)$. (G) Diarrhea $\left({ }^{* *} p<0.01\right)$. (H) Flatulence $\left({ }^{*} p<0.05\right)$. (I) Lachrymation $\left({ }^{*} p<0.05\right)$. (J) Anal sac repletion $\left({ }^{* *} p<0.01\right)$. The error bars are \pm the standard error of mean. Please click here to view a larger version of this figure. 


\section{Bark}

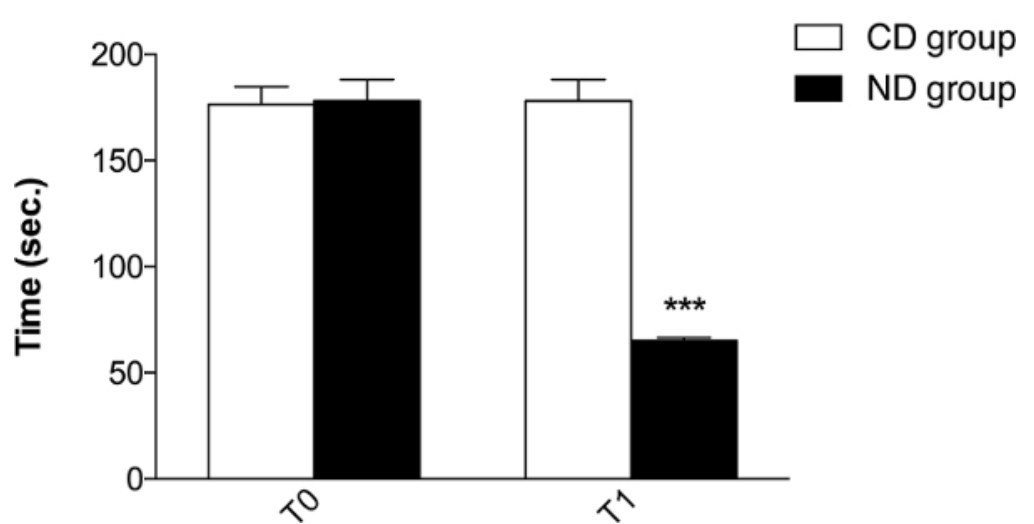

Figure 4. ND Reduces the Bark Time in Supplemented Dogs. Schematic representation of the mean time spent barking in dogs before (T0) and 10 days after $(T 1)$ SD and ND supplementation $\left({ }^{* * *} p<0.001\right)$. The error bars are \pm the standard error of mean. Please click here to view a larger version of this figure.

\begin{tabular}{|l|l|}
\hline Daily Ratio & \multicolumn{2}{|l|}{} \\
\hline Weight $(\mathrm{kg})$ & Amount $(\mathrm{g})$ \\
\hline $1-10$ & $30-180$ \\
\hline $11-20$ & $190-300$ \\
\hline $21-35$ & $310-455$ \\
\hline $36-50$ & $465-595$ \\
\hline
\end{tabular}

Table 1. Daily Amount of Food Provided to the Dogs.

\section{Discussion}

Both SD and ND were two commercially available diets that completely fulfill the recommendations for protein, carbohydrate, and fat content according to the nutritional guidelines for complete and complementary pet food. However, in the ND, nutraceutical substances, such as Punica granatum $(0.0457 \%)$, Valeriana officinalis $(0.026 \%)$, Rosmarinus officinalis $(0.000044 \%)$, Tilia spp $(0.0635 \%)$, tea extract $(0.031 \%)$, and Ltryptophan $(0.0329 \%)$, were added. It is noteworthy that this clinical evaluation was inspired by a previous trivial evaluation where 2 dogs with evident behavioral symptoms mainly ascribed to generalized anxiety showed significant improvements after 3 days of ND supplementation. Here, we successfully used the same ND with 24 dogs presenting behavioral symptoms mainly ascribed to generalized anxiety.

The only critical step that occurred in the protocol was related to the Wi-Fi connection. In some boxes, the sensor signal did not reach the WiFi station and therefore did not provide any data on the activity of the dogs. Thus, a Wi-Fi range extender was used to completely cover the distance between those boxes and the Wi-Fi station. Many studies have been performed to validate the usefulness of small, lightweight, motionsensing accelerometers for pets ${ }^{44}$ and humans ${ }^{46-54}$. The sensor used in this clinical evaluation presents some limitations with respect to the goldstandard method of using a video camera ${ }^{55,56}$, such as a lack of specificity in distinguishing rest activity from sleep and general motion from an anxiety-related one. On the other hand, the sensor allows for the easy and fast detection of movement, as well as the ability to monitor daily improvements by means of a mobile phone app. Moreover, with respect to the other commercially available devices, this new sensor has a lower weight and price, can be worn by a dog of any weight, and has a long-lasting ( 14 days) battery life. Moreover, because of the Wi-Fi station, it does not require the owner to be close to the dog while it is registering the improvements ${ }^{57-59}$. In fact, after registration with the website of the product company, the station can gather and store information that can be seen either on a computer or on a mobile device, even at a long distance (i.e., beyond the Bluetooth and Wi-Fi range). Possible further applications of this sensor will be the monitoring of excessive movement in dogs affected by separation anxiety ${ }^{47-54}$, abnormal repetitive behaviors ${ }^{60}$, or narcolepsy ${ }^{61}$ once left alone at home.

Our results pave the way for a different short-term approach to manage dogs with behavioral symptoms mainly ascribed to generalized anxiety disorders, allowing the owner to re-establish a mutual attachment relationship with the dog. In conclusion, a better understanding of dog behavior, both by pet owners and by behavioral experts able to recognize behavioral and clinical symptoms related to generalized anxiety, might be coupled with a specific diet in order to ensure a better quality of life for the animals.

\section{Disclosures}

The authors have nothing to disclose. 


\section{Acknowledgements}

This review was not supported by grants. We thank Sanypet Forza 10 USA Corp. Orlando, FL, USA for kindly providing the ND used in this study.

\section{References}

1. Ibáñez Talegón, M., Anzola Delgado, B. in http://www.intechopen.com/books/anxiety-disorders/anxiety-disorders-in-dogs1. ed Prof. Vladimir Kalinin (2011).

2. Houpt, K. A., Honig, S. U., Reisner, I. R. Breaking the human-companion animal bond. J Am Vet Med Assoc. 208, 1653-1659 (1996).

3. Overall, K. L. Clinical behavioral medicine for small animals. xvi + 544, Mosby-Year Book, Inc., (1997).

4. Brousset Hernández-Jáuregui, D. M., Galindo Maldonado, F., Valdez Pérez, R. A., Romano Pardo, M., Schuneman de Aluja, A. Cortisol en saliva, orina y heces: evaluación no invasiva en mamíferos silvestres. Vet Méx. 36, 325-337 (2005).

5. Flannigan, G., Dodman, N. H. Risk factors and behaviors associated with separation anxiety in dogs. J Am Vet Med Assoc. 219, 460-466 (2001).

6. Pageat, P. Patología del comportamiento del perro. Pulso, (2000).

7. Serpell, J. The Domestic Dog: Its Evolution, Behaviour and Interactions with People. Cambridge University Press, (1995).

8. Overall, K. L. Pharmacologic treatments for behavior problems. Vet Clin North Am Small Anim Pract. 27, 637-665 (1997).

9. Riaz, A, Khan, R. A. Effect of Punica Granatum on behavior in rats. Afr J Pharm Pharmacol. 8, 1118-1126 (2014).

10. Das. S, Sarma, P. A study on the anticonvulsant and anti anxiety activity of ethanolic extract of Punica granatum Linn. Int. J. Pharm. Scie. 6, 389-392 (2014).

11. Sudati, J. H. et al. In vitro Antioxidant Activity of Valeriana officinalis Against Different Neurotoxic Agents. Neurochem Res. 34, 1372-1379 (2009).

12. Hattesohl, M., Feistel, B., Sievers, H., Lehnfeld, R., Hegger, M., Winterhoff, H. Extracts of Valeriana officinalis L. s.I. show anxiolytic and antidepressant effects but neither sedative nor myorelaxant properties. Phytomedicine. 15, 2-15 (2008).

13. Wang, J. et al. Chemical Analysis and Biological Activity of the Essential Oils of Two Valerianaceous Species from China: Nardostachys chinensis and Valeriana officinalis. Molecules. 15, 6411-6422 (2010).

14. Carlini, E. A. Plants and the central nervous system. Pharmacol Biochem Behav. 75, 501-512 (2003).

15. Ulbricht, C. et al. An Evidence-Based Systematic Review of Rosemary (Rosmarinus officinalis) by the Natural Standard Research Collaboration. J Diet Suppl. 7 (2010).

16. Machado, D. G. et al. Antidepressant-like effect of the extract of Rosmarinus officinalis in mice: Involvement of the monoaminergic system Prog Neuropsychopharmacol Biol Psychiatry. 33 642-650 (2009).

17. Viola, H. et al. Isolation of pharmacologically active benzodiazepine receptor ligands from Tilia tomentosa (Tiliaceae). J Ethnopharmacol. 44, 47-53 (1994).

18. Coleta, M., Campos, M. G., Cotrim, M. D., Proenca da Cunha, A. Comparative evaluation of Melissa officinalis L., Tilia europaea L., Passiflora edulis Sims. and Hypericum perforatum L. in the elevated plus maze anxiety test. Pharmacopsychiatry. 34 Suppl 1, S20-21 (2001).

19. Juneja, L. R., Chu, D.-C., Okubo, T., Nagato, Y, Yokogoshi, Y. L-theanine-a unique amino acid of green tea and its relaxation effect in humans. Trends Food Sci Technol. 10, 199-204 (1999).

20. Miodownik, C. et al. Serum Levels of Brain-Derived Neurotrophic Factor and Cortisol to Sulfate of Dehydroepiandrosterone Molar Ratio Associated With Clinical Response to L-Theanine as Augmentation of Antipsychotic Therapy in Schizophrenia and Schizoaffective Disorder Patients. Clin Neuropharmacol. 34, 155-160 (2011).

21. Delgado, P. L. et al. Tryptophan-depletion challenge in depressed patients treated with desipramine or fluoxetine: implications for the role of serotonin in the mechanism of antidepressant action. Biol Psychiatry. 46, 212-220 (1999).

22. Delgado, P. L., Charney D. S., Price, L. H., Aghajanian, G. K., Landis, H., Heninger, G. R. Serotonin function and the mechanism of antidepressant action. Reversal of antidepressant-induced remission by rapid depletion of plasma tryptophan. Arch Gen Psychiatry. 47, 411-418 (1990).

23. Valvassori, S. S. et al. Sodium butyrate has an antimanic effect and protects the brain against oxidative stress in an animal model of mania induced by ouabain. Psychiatry Res. 235, 154-9 (2015).

24. Stoll, A. L. et al. Omega 3 Fatty Acids in Bipolar Disorder: A Preliminary Double-blind, Placebo-Controlled Trial FREE. Arch Gen Psychiatry. 56, 407-412 (1999).

25. Owen, C., Rees, A. M., Parker, G. The role of fatty acids in the development and treatment of mood disorders. Curr Opin Psychiatry. 21, 19-24 (2008)

26. Kilkenny, C., Browne, W. J., Cuthi, I., Emerson, M., \& Altman, D. G. Improving bioscience research reporting: the ARRIVE guidelines for reporting animal research. Vet Clin Pathol. 41, 27-31 (2012).

27. Di Cerbo, A., Palmieri, B., Chiavolelli, F., Guidetti, G., Canello, S. Functional foods in pets and humans. Intern J Appl Res Vet Med. 12, 192-199 (2014).

28. Hovatta, I., Juhila, J., Donner, J. Oxidative stress in anxiety and comorbid disorders. Neurosci Res. 68, 261-275 (2010).

29. Di Cerbo, A. et al. Toxicological Implications and Inflammatory Response in Human Lymphocytes Challenged with Oxytetracycline. $J$ Biochem Mol Toxicol. 30, 170-177 (2016).

30. Odore, R. et al. Cytotoxic effects of oxytetracycline residues in the bones of broiler chickens following therapeutic oral administration of a water formulation. Poult Sci. 94, 1979-1985 (2015).

31. Di Cerbo, A. et al. Clinical evaluation of an antiinflammatory and antioxidant diet effect in 30 dogs affected by chronic otitis externa: preliminary results. Vet Res Commun. 40, 29-38 (2016).

32. Di Cerbo, A., Canello, S., Guidetti, G., Laurino, C., Palmieri, B. Unusual antibiotic presence in gym trained subjects with food intolerance; a case report. Nutr Hosp. 30, 395-398 (2014). 
33. Kimera, Z. I. et al. Determination of oxytetracycline residues in cattle meat marketed in the Kilosa district, Tanzania. Onderstepoort $J$ Vet Res. 82, 911 (2015).

34. Chuah, L. O., Effarizah, M. E., Goni, A. M., Rusul, G. Antibiotic Application and Emergence of Multiple Antibiotic Resistance (MAR) in Global Catfish Aquaculture. Curr Environ Health Rep. 3, 118-127 (2016).

35. Chopra, I., \& Roberts, M. Tetracycline antibiotics: mode of action, applications, molecular biology, and epidemiology of bacterial resistance. Microbiol Mol Biol Rev. 65, 232-260 (2001).

36. Milch, R. A., Rall, D. P., Tobie, J. E. Bone localization of the tetracyclines. J Natl Cancer Inst. 19, 87-93 (1957).

37. Rivera, J. A., Sebranek, J. G., Rust, R. E. Functional properties of meat by-products and mechanically separated chicken (MSC) in a highmoisture model petfood system. Meat Sci. 55, 61-66 (2000).

38. Felger, J. C., Lotrich, F. E. Inflammatory cytokines in depression: neurobiological mechanisms and therapeutic implications. Neuroscience. 246, 199-229 (2013).

39. Lee, C.-J., Chen, L.-G., Liang, W.-L., Wang, C.-C. Anti-inflammatory effects of Punica granatum Linne invitro and in vivo. Food Chem. 118, 315-32 2 (2010).

40. Wojdyło, A., Oszmiański, J., Czemerys, R. Antioxidant activity and phenolic compounds in 32 selected herbs. Food Chem. 105, 940-949 (2007).

41. Erkan, N., Ayranci, G., Ayranci, E. Antioxidant activities of rosemary (Rosmarinus Officinalis L.) extract, blackseed (Nigella sativa L.) essential oil, carnosic acid, rosmarinic acid and sesamol. Food Chem. 110, 76-82 (2008).

42. Speisky, H., Rocco, C., Carrasco, C., Lissi, E. A., Lopez-Alarcon, C. Antioxidant screening of medicinal herbal teas. Phytother Res. 20, 462-467 (2006).

43. Katiyar, S. K., Elmets, C. A. Green tea polyphenolic antioxidants and skin photoprotection (Review). Int J Oncol. 18, 1307-1313 (2001).

44. Re, S., Zanoletti, M., Emanuele, E. Aggressive dogs are characterized by low omega-3 polyunsaturated fatty acid status. Vet Res Commun. 32, 225-230 (2008).

45. Colter, A. L., Cutler, C., Meckling, K. A. Fatty acid status and behavioural symptoms of attention deficit hyperactivity disorder in adolescents: a case-control study. Nutr J. 7, 8 (2008).

46. Lascelles, B. D., Hansen, B. D., Thomson, A., Pierce, C. C., Boland, E., Smith, E. S. Evaluation of a digitally integrated accelerometer-based activity monitor for the measurement of activity in cats. Vet Anaesth Analg. 35, 173-183 (2008).

47. Brown, D. C., Boston, R. C., Farrar, J. T. Use of an activity monitor to detect response to treatment in dogs with osteoarthritis. J Am Vet Med Assoc. 237, 66-70 (2010).

48. Yam, P. S. et al. Validity, practical utility and reliability of Actigraph accelerometry for the measurement of habitual physical activity in dogs. $J$ Small Anim Pract. 52, 86-91 (2011).

49. Michel, K. E, Brown, D. C. Determination and application of cut points for accelerometer-based activity counts of activities with differing intensity in pet dogs. Am J Vet Res. 72, 866-870 (2011).

50. Hansen, B. D., Lascelles, B. D., Keene, B. W., Adams, A. K., Thomson, A. E. Evaluation of an accelerometer for at-home monitoring of spontaneous activity in dogs. Am J Vet Res. 68, 468-475 (2007)

51. Moreau, M., Siebert, S., Buerkert, A., Schlecht, E. Use of a tri-axial accelerometer for automated recording and classification of goats' grazing behaviour. Appl Anim Behav Sci. 119, 158-170 (2009).

52. Yamada, M., Tokuriki, M. Spontaneous activities measured continuously by an accelerometer in beagle dogs housed in a cage. $J$ Vet Med Sci. 62, 443-447 (2000).

53. Preston, T., Baltzer, W., Trost, S. Accelerometer validity and placement for detection of changes in physical activity in dogs under controlled conditions on a treadmill. Res Vet Sci. 93, 412-416 (2012).

54. Yashari, J. M., Duncan, C. G., Duerr, F. M. Evaluation of a novel canine activity monitor for at-home physical activity analysis. BMC Vet Res. 11, 146 (2015).

55. Troiano, R. P., Berrigan, D., Dodd, K. W., Mâsse, L. C., Tilert, T., McDowell, M. Physical activity in the United States measured by accelerometer. Med Sci Sports Exerc. 40, 181-188 (2008).

56. Stratford, P. W., Kennedy, D. M. Performance measures were necessary to obtain a complete picture of osteoarthritic patients. $J$ Clin Epidemiol. 59, 160-167 (2006).

57. Parthasarathy, V., Crowell-Davis, S. L. Relationship between attachment to owners and separation anxiety in pet dogs (Canis lupus familiaris). J Vet Behav Clin Appl Res. 1, 109-120 (2006).

58. Palestrini, C., Minero, M., Cannas, S., Rossi, E., Frank, D. Video analysis of dogs with separation-related behaviors. Appl Anim Behav Sci. 124, 61-67 (2010)

59. Mills, D. S., Ramos, D., Estelles, M. G., Hargrave, C. A triple blind placebo-controlled investigation into the assessment of the effect of Dog Appeasing Pheromone (DAP) on anxiety related behaviour of problem dogs in the veterinary clinic. Appl Anim Behav Sci. 98, 114-126 (2006).

60. Irimajiri, M., Crowell-Davis, S. L. Animal behavior case of the month. Separation anxiety. J Am Vet Med Assoc. 245, 1007-1009 (2014).

61. Tynes, V. V., Sinn, L. Abnormal repetitive behaviors in dogs and cats: a guide for practitioners. Vet Clin North Am Small Anim Pract. 44, 543-564 (2014). 